\title{
IMAGING BIOMARKERS OF ANTI-EPILEPTIC DRUG ACTION: INSIGHTS FROM MAGNETIC RESONANCE IMAGING AND SPECTROSCOPY
}

Lorenzo Caciagli $^{1,2}$, Fenglai Xiao ${ }^{1,2,3}$, Britta Wandschneider ${ }^{1,2}$, Matthias J. Koepp ${ }^{1,2}$

${ }^{I}$ Department of Clinical and Experimental Epilepsy, UCL Institute of Neurology, Queen Square, London, United Kingdom

${ }^{2}$ MRI Unit, Epilepsy Society, Chalfont St Peter, Buckinghamshire, United Kingdom

${ }^{3}$ Department of Neurology, West China Hospital, Sichuan University, Chendgu, Sichuan, People's Republic of China

\section{Correspondence to}

Prof. Matthias Koepp, MD PhD

Department of Clinical and Experimental Epilepsy

UCL Institute of Neurology

Queen Square, London WC1N 3BG, UK

MRI Unit, National Society for Epilepsy

Chalfont St Peter, Buckinghamshire, SL9 0RJ

Tel. 01494601300

Fax 0149487464

E-mail: m.koepp@ucl.ac.uk 


\begin{abstract}
Background: Approximately one third of patients with epilepsy are refractory to medical treatment. Adverse effects associated with anti-epileptic drugs (AEDs) are considered to affect quality of life often more than the seizures themselves. Neuroimaging techniques, in particular magnetic resonance imaging (MRI), have proven instrumental in clinical decision making in relation to epilepsy surgery, but may also provide further insights into the mechanisms underlying treatment response and side effects associated with AEDs.
\end{abstract}

Objective and Method: We searched PubMed and Ovid Medline databases for original articles and reviews published in the last two decades, which addressed the effects of AEDs on MRI and magnetic resonance spectroscopy (MRS) measures.

Results: The majority of investigations implemented task-based fMRI, and probed the influence of widely used anti-epileptic drugs on tasks assessing language, executive functions and emotion recognition. Collectively, MRI is able to detect reproducible AED-related effects on regions and networks relevant to disease pathomechanisms, and so elucidates the anatomofunctional substrates of cognitive side effects. MRS analyses shed light on the molecular correlates of AED action, and may provide indicators of treatment response.

Conclusion: MRI and MRS have considerably improved our understanding of the effects of AEDs at a regional and network level, and provide biomarkers with potential to improve routine clinical decision making in epilepsy.

\title{
KEYWORDS
}

Antiepileptic drug, MRI, functional MRI, MR spectroscopy, biomarker, cognitive side effects, drug response 


\section{INTRODUCTION}

Epilepsy is characterized by the neurobiological, cognitive, psychological, and social consequences of this condition, which are confounded by treatment. . Refractoriness to pharmacological treatment, termed as 'drug-resistance', is estimated to occur in about $30 \%$ of patients with epilepsy (Sander, 2003; Kwan et al., 2010), despite significant advances in drug development over the last two decades, leading to more than twenty anti-epileptic drugs (AEDs). Moreover, AED-correlated adverse effects play a role in determining treatment failures, are associated with morbidity and mortality, and can considerably affect quality of life of people with epilepsy (Perucca and Gilliam, 2012).

Neuroimaging, in particular magnetic resonance imaging (MRI), contribute to diagnosis and clinical management (Bernasconi et al., 2011; Duncan et al., 2016). Advanced MRI techniques prove useful in characterising functional and structural brain networks in vivo (Honey et al., 2007; Sporns, 2012; Bernhardt et al., 2013; Caciagli et al., 2014), and provide objectively measurable traits, which exhibit validity as indicators of biological processes as well as high test-retest reliability, thus fulfilling the defining criteria of a biomarker (??? 2001; Koepp, 2016). Given their ability to identify anatomo-functional markers of disease effects, MRI techniques have also been utilised to detect measurable responses to pharmacological interventions, with the aim to optimise medication choices at the individual level, and aid rationalisation of polytherapy strategies (van Veenendaal et al., 2015; Wandschneider and Koepp, 2016). In this context, functional MRI (fMRI) investigations shed light on regional and network-level dynamics, and may prove particularly sensitive in unveiling drug-related effects (Nathan et al., 2014; Beltramini et al., 2015). As well as identifying markers of treatment response and dose-response relationships, fMRI also conveys the possibility to address a variety of cognitive functions via specific tasksrelevant to epilepsy, which is accompanied by cognitive comorbidities. with AEDs often causing additional cognitive side effects (Ortinski and Meador, 2004; Hermann et al., 2008; Mula and Trimble, 2009).

In this review, we will summarise the most recent studies investigating the effects of common AEDs on MRI parameters. We will focus first on pharmacological fMRI (phMRI) studies, though relevant insights from other, structural MRI and magnetic resonance spectroscopy (MRS) metrics will also be discussed. to probe the neurochemical underpinnings of AED effects in vivo. 


\section{MRI BIOMARKERS OF AED ACTION: AN OVERVIEW}

Ph-MRI study designs provide imaging correlates of AED effects. While structural MRI metrics evaluate the integrity of grey matter structures and of the interconnecting white matter tracts, fMRI conveys an indirect measure of neuronal activity via the Blood-Oxygen-LevelDependent (BOLD) contrast, which arises from localised changes in the ratio between oxygenated and deoxygenated haemoglobin owing to metabolic demands driven by neuronal activity (Ogawa et al., 1990; Logothetis et al., 2001). Functional MRI data can be acquired while participants engage in specifically designed paradigms, which generate activation and deactivation patterns pertaining to brain regions subserving language, executive functions, episodic memory and sensory-motor processing (Rao et al., 1993; Owen et al., 2005; Kim, 2011; Price, 2012). In addition, "resting-state" fMRI sequences are commonly used, which specifically address spontaneous fluctuations of BOLD signal occurring during rest (i.e., task free conditions)(Fox and Raichle, 2007), and identify stable and reproducible sets of regions with strongly correlated signal time-courses, and high correspondence with task-implicated systems (Damoiseaux et al., 2006; Biswal et al., 2010).

Ph-MRI studies have thus far been conducted mostly via task-based fMRI. Here, effects associated with pharmacological agents are predominantly shown as interaction effects, whereby a comparison takes place between activation patterns in subjects on drugs against placebo, or against subjects not taking the drug (Mehta and O'Daly, 2011). Randomised, double-blind, within-subject designs have also been implemented, though predominantly for assessments targeting psychiatric populations (Hafeman et al., 2012). The impact of drugs can be examined at a regional as well as at a network level, formalising connectivity properties via measures of statistical dependency between spatially separate BOLD time courses (Friston, 2011). Compared to other in vivo techniques, including positron emission tomography (PET), the advantage of pharmacofMRI is to potentially assess large-scale systems providing snapshots of network architecture across multiple scales and during different tasks, irrespective of the pharmacodynamic properties of a given compound (Borsook et al., 2006; Nathan et al., 2014). This resulted in pharmacofMRI to be used not only to investigate already marketed drugs, but also in the context of drug discovery (Nathan et al., 2014). 
This review summarises pharmaco-fMRI studies of the most frequently prescribed AEDs. Where appropriate, analyses investigating the impact of AEDs on structural MRI metrics are also considered. (Hafeman et al., 2012).

\subsection{TOPIRAMATE AND ZONISAMIDE}

Topiramate (TPM) is currently approved for the treatment of epilepsy, in monotherapy as well as an add-on compound, and is additionally licensed for migraine prophylaxis (Bootsma et al., 2004; Mula, 2012). Despite the efficacy of TPM across disease entities, there appears to be an association between its usage and neurocognitive impairment, both in healthy volunteers as well as patients with epilepsy and migraine disorders. Dysfunction appears to affect several domains, including sustained attention, psychomotor speed, working-memory, as well as expressive language skills, addressed by verbal fluency tasks (Martin et al., 1999; Thompson et al., 2000; Lee et al., 2003; Mula et al., 2003; Meador et al., 2005).

The frequent incidence of cognitive impairment accompanying TPM treatment has prompted investigations into its pathophysiological underpinnings via cognitive functional MRI paradigms. TPM is the most frequently studied drug by pharmaco-fMRI, though in the majority with small participant samples (Beltramini et al., 2015; Wandschneider and Koepp, 2016). Given the impact on language skills, several studies utilised expressive language fMRI tasks, such as verbal fluency tasks, during which participants are usually required to generate words starting with specific letters (phonemic fluency). As these paradigms often alternate active with baseline blocks of low-demand information processing (e.g., cross-hair fixation), the activation maps generally reveal areas involved in speech production (dominant inferior frontal cortex) along with regions more broadly subserving attention, task execution and working memory, including dorso-lateral frontal, medial frontal and posterior parietal cortices. Implementing a verbal fluency task, Jansen and collaborators first revealed disrupted activation of inferior frontal and medial prefrontal cortices in five patients with focal epilepsy taking TPM against ten subjects with epilepsy not on TPM, along with variable reconfiguration of occipito-parietal activations (Jansen et al., 2006). Another verbal fluency study analysed ten patients on TPM for migraine prophylaxis, five of whom subjectively reported cognitive dysfunction. Compared with controls, attenuated activation of task-related frontal language areas was demonstrated in the subgroup with subjective cognitive difficulties, whereas subjects on TPM without cognitive deficits showed increased activation of language areas, interpreted by the authors as a potential 
compensatory mechanism (De Ciantis et al., 2008). A subsequent verbal fluency fMRI study on patients with epilepsy implemented a cross-sectional and a longitudinal component. For the cross-sectional arm, Yasuda et al. recruited patients with frontal lobe epilepsy (FLE) and demonstrated impaired task-related deactivation of midline fronto-parietal default mode areas, required for effective performance of cognitive tasks (Weissman et al., 2006; Sonuga-Barke and Castellanos, 2007), in patients on TPM compared to patients not taking TPM and healthy controls (Yasuda et al., 2013). This imaging trait interestingly displayed a highly significant positive correlation with drug dose. In the longitudinal component of the study, one TPM-off and one TPM-on task runs were compared for four FLE patients and two healthy volunteers, corroborating cross-sectional evidence of task-related failure to deactivate default mode areas during the TPM-on condition. In addition, qualitatively reduced activation of frontal language areas was also documented on TPM. Additional evidence of impaired deactivation of default mode areas during expressive language came from a subsequent study utilising a verb generation paradigm, asking participants to view concrete nouns and generate semanticallycorrelated verbs (Tang et al., 2016). The investigators recruited a mixed group of 12 patients with focal epilepsy taking TPM as add-on therapy, six of whom subsequently discontinued it on clinical grounds. Overall, patients on TPM showed reduced task-related deactivation within anterior and posterior default mode areas compared with controls. In those who discontinued TPM, analysis of task-related maps post-withdrawal unveiled increased activation of right middle frontal and left superior occipital gyrus, as well as enhanced deactivation of left parahippocampal gyrus and bilateral posterior cingulate cortex.

While the above detailed studies addressed expressive language, Szaflarski and collaborators implemented a semantic decision/tone decision fMRI task (Szaflarski and Allendorfer, 2012) to map the speech comprehension system, which extends to a considerable proportion of the prefrontal cortex, temporal areas (lateral and ventral, not reliably activated by verbal fluency tasks), as well as angular and posterior cingulate gyrus (Binder et al., 2008). Distinct activation patterns emerged when comparing left TLE patients on- versus off-TPM, and right TLE onand off-TPM. Among patients with left-sided foci, those on TPM exhibited reduced activation of the left superior temporal gyrus and hyperactivation of the left anterior cingulate cortex compared with those off TPM. Right TLE patients on TPM presented with decreased activation of the inferior frontal gyrus and enhanced recruitment of bilateral superior frontal and left temporo-occipital areas. Further, greater prevalence of atypical language representation was noticed for both TLE patients on TPM than those off -TPM (Szaflarski and Allendorfer, 2012). 
In summary, fMRI studies addressing the impact of TPM on language networks utilised a variety of tests, examined different and heterogeneous subject categories, and generally relied on small sample sizes. Nonetheless, converging evidence across investigations points to disrupted activation of task-relevant fronto-temporal cortices, as well as impaired de-activation of task-negative regions, predominantly represented by the default mode network. The potential mechanisms leading to such effects have been speculated upon. The presence of a sulphonamide functional group provides TPM with carbonic anhydrase inhibition properties (Leniger et al., 2004), which may influence the regional BOLD contrast measured by fMRI. This was demonstrated for acetazolamide, a widely utilised carbon anhydrase inhibitor, shown to induce increases in cerebral perfusion while not affecting oxygen consumption (Bruhn et $a l ., 1994)$. Thus, an altered ratio between rest and activation BOLD contrast may take place, represented by an attenuated signal change in response to an increase in activity of a certain area, as shown for the visual cortex during photic stimulation (Bruhn et al., 1994). While this phenomenon may prominently occur in brain areas recruited during a task, and might explain the attenuated activation of task-positive areas observed by fMRI studies, an elucidation of its influence on task-related deactivations and their spatial distribution appears less intuitive. Moreover, TPM is about 10 to 100 times less potent as carbon anhydrase inhibitor than acetazolamide (Shank et al., 1994) and exhibits multifaceted pharmacodynamic properties, including blockade of voltage-gated sodium channels, L-type calcium channels, AMPA/kainate subtypes of glutamate receptors as well as potentiation of $\mathrm{GABA}_{\mathrm{A}}$-mediated neurotransmission (Perucca, 1997; Mula, 2012), whose effect on fMRI activation patterns also needs to be taken into account during data interpretation.

Valuable insights may come from investigations addressing zonisamide, an AED utilised to treat focal and generalised epilepsies and associated with a profile of cognitive side effects which is comparable, though possibly more moderate, with that detailed for TPM (Ojemann $e t$ al., 2001; Mula and Trimble, 2009). Mechanisms of action of zonisamide include blockade of voltage-sensitive sodium channels, T-type calcium channels, modulation of dopaminergic and serotoninergic transmission as well as a neuroprotective effect from free-radical damage (Leppik, 2004; Patsalos, 2005). As described for TPM, zonisamide also exhibits a sulpha moiety and weak carbon anhydrase inhibition properties, estimated as 100-1000 times lower than acetazolamide (Leppik, 2004). A recent electroencephalography (EEG) study on individuals taking zonisamide for recent-onset epilepsy reported reduced current source density of beta frequencies for areas implicated in language and working memory, such as 
inferior and middle frontal cortices, anterior cingulate gyri and inferior parietal lobules (Kwon and Park, 2013). In a retrospective analysis of verbal fluency fMRI, comparing patients taking Topiramate ( $\mathrm{n=32}$ ), Zonisamide (51) and Levetiracetam (62) (Wandschneider et al., 2017), we observed a similar influence of zonisamide and TPM on fronto-parietal cognitive networks in patients with focal epilepsy, as measured by verbal fluency fMRI. However, impaired taskrelated deactivation of default mode areas was described for the TPM group only, and not in individuals on zonisamide. These results provide evidence for medication-specific effects, substantially advancing our understanding of the impact of distinct AEDs on the architecture of cognitive networks. From a mechanistic viewpoint, it would remain to be established whether distinct effects of TPM and zonisamide may be ascribed to differences in carbonic anhydrase inhibition activity, which appears to be higher for topiramate. In this regard, characterising the influence of acetazolamide on cognitive performance and activation patterns during linguistic and executive tasks may prove useful.

\subsection{CARBAMaZePine/OXCARbaZePine}

Carbamazepine (CBZ) represents one of the first-generation AEDs and, along with its derivative oxcarbazepine (OXC), mainly owes its seizure suppressant activity to the inhibition of voltage-dependent sodium channels (Ambrosio et al., 2002). Interaction with potassium and L-type calcium channels, GABA $\mathrm{A}_{\mathrm{A}}$ receptors as well as adenosine binding sites are also reported (Liu et al., 2006). CBZ represents the first drug to be investigated within an fMRI study setting. Jokeit and colleagues analysed a visuo-spatial memory retrieval task to activate mesiotemporal structures via mental navigation through a familiar route, in 21 individuals with drug-resistant TLE. An inverse correlation was detected between fMRI activations within mesiotemporal lobes and CBZ serum levels, with no additional influence exerted by lateralisation of epilepsy nor further contribution of CBZ-epoxide serum level to data explanation (Jokeit et al., 2001). This provides evidence for a CBZ-related attenuation of mesiotemporal activations with a linear dose-response relationship. More recently, a resting-state fMRI analysis on patients with TLE compared a subgroup treated with CBZ/OXC against participants taking other AEDs (Haneef et al., 2015), and characterised the organisational properties of functional networks via a graph-theoretical approach (Bullmore and Sporns, 2009). Patients treated with OXC/CBZ differed from those on other AEDs with respect to "betweenness centrality", a metric utilised to identify 'hubs', nodes exhibiting high connectional weighs within the architecture of a 
network, and prominent roles in ensuring connectivity between distant brain areas. The authors detected variations of betweenness centrality in several nodes, including decreases in mesiotemporal and orbitofrontal areas, anterior cingulate cortices, angular gyrus, thalamus and putamen, co-existing with increases in posterior cingulate, insular, lateral temporal, posterior parietal and temporo-occipital cortices (Haneef et al., 2015). On balance, these results may indicate redistribution of hub properties with more marked shifts from limbic to lateral cortical areas in TLE treated with CBZ/OXC. Interestingly, recent evidence from graph theoretical analyses on structural and functional MRI datasets from patients with TLEpoints to disrupted nodal topology of limbic subnetworks (Liao et al., 2010; Chiang et al., 2014; Bernhardt et al., 2015), along with major rearrangements of whole-brain network hubs, particularly involving para-limbic and lateral temporal cortices (Wang et al., 2014). Thus, CBZ/OXC may be influencing the topological properties of subnetworks specifically involved in disease pathomechanisms.

\subsection{LEVETIRACETAM}

Levetiracetam (LEV) is one of the most widely used AEDs, and received approval in numerous countries as monotherapy as well as add-on treatment for focal and generalised epilepsies. LEV binds to the synaptic vesicle protein SV2A, probably modulating synaptic neurotransmitter release (Lynch et al., 2004). In contrast to other AEDs, LEV has been repeatedly shown not to exert a detrimental impact on cognition, oror even amelioration of neuropsychological performance (Piazzini et al., 2006; Helmstaedter and Witt, 2008), depicting a more favourable profile than for other AEDs, including carbamazepine (Mecarelli et al., 2004; Meador et al., 2007; Helmstaedter and Witt, 2010). A recent EEG study provided evidence for multi-domain cognitive improvement along with accelerated background frequencies in drug-naïve epilepsy patients after treatment with LEV (Cho et al., 2012).

To characterise the imaging underpinnings of the effect of LEV on cognition, we utilised both a verbal and a visuo-spatial fMRI working memory task in a large cohort of 106 patients with TLE, 59 of whom treated with LEV, and with no between-group divergences regarding TPM or zonisamide co-medication. Compared with individuals not treated with LEV, patients on LEV exhibited increased task-related deactivations in the affected temporal lobe, namely in the left middle temporal gyrus in left TLE during the verbal working memory paradigm, and in the right hippocampus in right TLE during the visuo-spatial task. The latter effects occurred in a 
dose-dependent fashion, and were not evidenced when comparing patients treated with or without CBZ, or with and without lamotrigine (Wandschneider et al., 2014). Previous investigations characterising the functional architecture of working memory networks illustrated the association between attenuated activation of mesiotemporal structures and effective task performance (Dolcos and McCarthy, 2006; Cousijn et al., 2012). This may occur as part of a resource redistribution process from task-irrelevant to task-relevant regions, aiming to minimise interference (Anticevic et al., 2010; Cousijn et al., 2012). Interestingly, studies addressing the fMRI correlates of working memory in TLE detected failure to deactivate the hippocampus ipsilateral to the seizure focus compared with healthy controls (Stretton et al., 2012), in the context of morpho-functional connectivity derangements pointing to a disrupted segregation between task-negative (mesiotemporal) and task-positive (parietal) areas (Stretton et al., 2013). Consequently, the influence of LEV on working memory networks in TLE, described by Wandschneider and colleagues, can be interpreted as a restoration of fMRI activation patterns described for healthy controls and, therefore, regarded as favourable.

Further evidence of a normalising effect of LEV on mesiotemporal activation comes from functional imaging studies conducted on subjects with amnestic mild cognitive impairment (aMCI) (Bakker et al., 2015), in whom augmented task-related activations in mesiotemporal structures were documented during memory encoding tasks (Dickerson et al., 2004; Celone et al., 2006). Comparing a short treatment course with low-dose LEV versus placebo, Bakker and collaborators elegantly demonstrated a reversal of the aberrant activation patterns of dentate gyrus/CA3 hippocampal sub-regions and entorhinal cortex to levels normally observed in healthy controls, accompanied by a significant improvement in task performance (Bakker et al., 2012; Bakker et al., 2015).

A recent analysis on patients with benign epilepsy with centro-temporal spikes (BCETS) documented an effect of LEV on the regional homogeneity (ReHo) of fMRI resting-state signal, which addresses the local synchronisation of fMRI time-series across a set of neighbouring voxels (Zang et al., 2004). As different from drug-naïve individuals, reduced ReHo was detected in patients on LEV in areas implicated in the generation of interictal epileptic spikes, such as fronto-centro-temporal cortices, basal ganglia and thalamus. Contrasting ReHo patterns in patients on LEV against those treated with sodium valproate (discussed below) revealed spatial specificity of drug effects, with LEV affecting more prominently fronto-temporal cortices and caudate nuclei while exerting a less pronounced influence on thalamic activity. LEV also exerted dissociating effects on the covariance of 
thalamic and centro-temporal local fMRI metrics, shown instead as significantly correlated in drug-naïve patients and subjects treated with valproate (Zhang et al., 2016). Unfortunately, the absence of a healthy control cohort in the above study impedes establishing whether regional homogeneity patterns in LEV-treated patients may indicate a reversal to normal baseline values.

Collectively, evidence across different disorders suggests a disease-specific distribution of the effects of LEV, with predominant influence on subnetworks relevant to disease pathomechanisms. Its effects may further exhibit beneficial implications for cognitive performance, as demonstrated for conditions with impact on mesiotemporal function.

\subsection{SODIUM VALPROATE}

Sodium valproate (VPA) is a well-established broad-spectrum AED with efficacy to treat a multiplicity of focal and generalised epilepsy syndromes, is utilised as mood stabiliser for the treatment of bipolar disorder, and is also licenced for migraine prophylaxis (Perucca, 2002; Nalivaeva et al., 2009). VPA has long been regarded as a compound with diverse and complex mechanisms of action, which go beyond the potentiation of GABAergic neurotransmission and include attenuation of NMDA glutamate receptor activity, blockade of voltage-sensitive sodium channels, and modulation of dopaminergic and serotoninergic transmission (Loscher, 2002; Perucca, 2002). Furthermore, VPA exposure leads to extensive modifications of gene expression, with downstream influence on transcription regulation, signal transduction and cellular homeostasis, probably underlain by its histone deacetylase (HDAC) pan-inhibition properties (Rosenberg, 2007; Nalivaeva et al., 2009; Rosenzweig et al., 2012). There is also evidence of potential neuroprotective effects brought about by VPA through the modulation of signalling cascades involved in neuronal apoptosis, formation of neurofibrillary tangles and amyloid plaques (Tariot et al., 2002).

The effects of a two-week long VPA course on the activation patterns of spatial attention, working memory and verbal fluency tasks were recently addressed by an fMRI study on healthy volunteers. Analysing the magnitude of BOLD signal change pre- and postintervention across task-specific maps, Bell and collaborators observed attenuated activations for the spatial attention and word generation tasks in the VPA-treated cohort compared to subjects receiving placebo. ROI-based post-hoc analysis revealed changes to occur in the left 
lingual gyrus during the attention task, and to encompass the supplementary motor area for the verbal fluency task. On the other hand, individuals on two-week course of lithium presented with significantly reduced activations within working memory and verbal fluency maps, but not during the attention task (Bell et al., 2005). These findings may reflect a differential effect of VPA and lithium on cognitive fMRI activations, and point to the influence of VPA on expressive language and attention networks, with relative sparing of working memory. As neurobehavioral correlates were not provided, it remains undetermined whether such reconfigured patterns may be accompanied by performance changes. The above findings, however, may be in line with evidence from neuropsychological studies, collectively indicating a mild impact of VPA on measures of attention, psychomotor speed and executive functions (Thompson and Trimble, 1981; Meador et al., 2003; Mula and Trimble, 2009).

Variable results were obtained in pharmaco-fMRI studies probing VPA-related effects in disease entities. In a small sample of children with mood dysregulation and familial history of bipolar disorder, no significant activation changes during an emotion attribution task were detected after three months of treatment with VPA, compared to baseline pre-treatment data. (Chang et al., 2009). Investigations on patients with epilepsy, on the other hand, may suggest beneficial effects of VPA on fMRI activation maps.

Focusing on patients with juvenile myoclonic epilepsy (JME) and their un-affected siblings, we detected co-activation of motor and cognitive areas during a visuo-spatial working memory task in patients with JME and their siblings compared with controls, which may represent an imaging trait underlying the highly prevalent cognitively-triggered jerks (Wandschneider et al., 2012; Wolf et al., 2015; Koepp et al., 2016). In JME patients, we observed an inverse relationship between VPA dose and motor cortex activation, which is indicative of a "normalising effect" of VPA on the aberrant activation patterns documented for motor areas in JME (Vollmar et al., 2011). Structural and functional connectivity was augmented between motor areas and prefrontal cognitive networks (Vollmar et al., 2012), In line with the above, evidence of an influence of VPA on the excitability of the motor system was also provided by analyses implementing interleaved fMRI/transcranial magnetic stimulation (TMS). During classical stimulation of the motor hotspot, diffuse attenuation of TMS-correlated BOLD response was observed within several areas belonging to the motor system after a single dose of VPA compared with placebo (Li et al., 2010), along with reduction of effective connectivity between primary motor cortex and both premotor and supplementary motor areas ( $\mathrm{Li}$ et al., 2011). 
In subjects affected by BCETS, regional homogeneity of fMRI time courses was attenuated in regions demonstrated to be implicated in epileptic spike generation, including fronto-centrotemporal cortices as well as thalamus, in subjects treated with VPA compared with drug-naïve individuals. Attenuation of centro-temporal regional homogeneity also appeared dosedependent. Compared with LEV, the impact of VPA was more pronounced on thalamic activity and less evident for cortical regions. Further, subjects on VPA displayed preserved covariance of fMRI metrics between thalamus and centro-temporal cortices, possibly suggesting a balanced effect on both cortical and subcortical structures associated with VPA (Zhang et al., 2016).

Imaging correlates of VPA use have also been sought for by structural MRI studies, possibly following early case reports of reversible VPA-associated pseudoatrophy and neurobehavioral deterioration (Papazian et al., 1995; Guerrini et al., 1998). In small samples of young individuals with subsyndromal bipolar disorder and matched controls, Chang and colleagues described no evidence of changes in total brain or amygdala volume after a three-month course of VPA (Chang et al., 2009). Moreover, VPA-treated adult patients with bipolar disorder were shown to present with greater volumes of anterior and posterior cingulate cortices, which exhibited a trend-level negative correlation with symptom severity (Atmaca et al., 2007). On the other hand, recent longitudinal clinical trials evaluating the effectiveness of VPA for the treatment of agitation and psychosis in Alzheimer's disease observed accelerated rates of whole-brain and hippocampal volume loss, along with more rapid ventricular expansion compared to the placebo cohort (Fleisher et al., 2011; Tariot et al., 2011). These changes were also paralleled by faster cognitive decline over the first treatment year, as measured by the Mini-Mental State Examination (Fleisher et al., 2011). In young children (age 6-8) incurred in prenatal exposure to VPA, an analysis revealed augmented cortical thickness of the pars opercularis of the left inferior frontal gyrus and of the left medial occipital cortex, with loss of the expected right-to-left thickness asymmetry within inferior frontal cortices. Further, a trend towards a negative correlation was observed between left fronto-opercular thickness and a composite measure of verbal comprehension (Wood et al., 2014). This study provides an imaging correlate of neurobehavioral findings detailed by multiple investigations, linking in utero exposure to VPA with poorer cognitive outcomes across several domains, particularly verbal abilities (Adab et al., 2004; Gaily et al., 2004; Meador et al., 2009; Meador et al., 2013), along with increased likelihood of atypical language lateralisation (Meador et al., 2011; Nadebaum et al., 2011). A study by Pardoe and colleagues identifiedreductions in total whole 
brain volume, white matter volume as well as parietal lobe thickness in subjects treated with VPA compared with healthy controls and patients not on VPA. These findings were documented in two separate cohorts, one represented by subjects with drug-resistant focal epilepsy and another consisting of patients with childhood-onset seizures. Effects associated with VPA were thought to be reversible, as no differences could be detected between previous VPA users and patients who were never prescribed the drug (Pardoe et al., 2013). The biological meaning of reductions in parietal lobe thickness could not be inferred, owing to the clinical heterogeneity of the analysed subjects, the absence of direct correlations with cognitive measures and of details regarding potential differences in seizure control among patient groups. Regarding decreases in white matter volume, however, experimental and human studies point to an impact of VPA on late-differentiating oligodendrocytes, due to its action as histone deacetylase pan-inhibitor (Shen et al., 2008; Rosenzweig et al., 2012). This effect may influence myelination/re-myelination efficiency, leading to derangements in white matter architecture and particularly affecting later-myelinating plastic circuits, which play crucial roles in multi-modal associative processing, cognitive control and goal-directed behaviour (Rosenzweig et al., 2012).

On balance, recent investigations have considerably advanced our understanding of the functional and structural MRI correlates of VPA usage. Overall, results of the above detailed studies appear multifaceted. While some fMRI studies may suggest a beneficial influence of VPA, with reverberation on disease-specific subnetworks, morphometry analyses concordantly report detrimental effects correlated with VPA use across diverse conditions, each of which tapping into different life spans. Proved heterogeneity in the analysed cohorts, study design and confounding factors may be accounted for the wide spectrum of findings. It is also possible, however, that the pleiotropic pharmacodynamic of VPA might lead to unique phenotypes arising from specific interactions between disease- and drug-related effects, ultimately justifying marked variability of imaging features condition per condition.

\subsection{LAMOTRIGINE}

Lamotrigine (LTG) is a drug with broad spectrum efficacy, spanning from focal to generalised epilepsy syndromes and including bipolar disorder, owing to its mood stabilisation properties. Its principal mechanism of action is represented by the use- and voltage-dependent blockade of sodium channels (Cheung et al., 1992; Coulter, 1997), possibly accompanied by inhibition 
of voltage-sensitive calcium currents, anti-glutamatergic and neuroprotective effects (Ketter $e t$ al., 2003).

Though a variety of research groups set out to investigate LTG-associated functional imaging markers, no studies at present recruited patients with epilepsy. Li and collaborators carried out a series of investigations aiming to establish the influence of LTG on fMRI activation patterns during motor and prefrontal TMS in healthy volunteers. As previously detailed for VPA, LTG was also shown to diffusely attenuate TMS-induced BOLD response over several areas of the motor system (Li et al., 2004; Li et al., 2010), and to impact effective connectivity between primary motor cortex and both premotor and supplementary motor areas (Li et al., 2011) compared with placebo. LTG-specific effects, however, emerged during prefrontal TMS, which generally elicits the activation of medial prefrontal as well as primary sensory areas. TMS-correlated fMRI activations for the LTG condition encompassed broader areas, with significantly increased recruitment of ipsilateral hippocampus, medial frontal, anterior cingulate and orbitofrontal cortices compared to placebo (Li et al., 2004; Li et al., 2010). This pattern was not detected for VPA, which caused attenuated activation of all target regions of prefrontal TMS when compared against placebo, and also of the above mentioned limbic areas, when directly compared with LTG (Li et al., 2010). Moreover, LTG was associated with increases in TMS-correlated effective connectivity between ipsilateral dorsolateral prefrontal and anterior cingulate cortex, while the same findings could not be detailed for VPA (Li et al., 2011). As limbic hyperactivation, frontal hypoactivation and dysfunctional fronto-cingulate connectivity have been implicated in the pathophysiology of major depression and bipolar disorder (Schlosser et al., 2008; Sheline et al., 2010; Kupferschmidt and Zakzanis, 2011; Pizzagalli, 2011; Strakowski et al., 2012), these findings provide support to the view that LTG may influence activity and connectional properties of brain areas relevant to disease pathophysiology. Indeed, several analyses focused on verbal working memory and facial emotion recognition fMRI in individuals with bipolar disorders. Haldane and colleagues report increased recruitment of task-relevant areas, such as dorso-lateral prefrontal, medial frontal and anterior cingulate cortices, after a six-week course of LTG (Haldane et al., 2008). In paediatric patients, reduced activation of the right amygdala during negative facial emotion recognition was detected after 8 weeks of treatment with LTG, and was positively related to improvements in depressive symptoms (Chang et al., 2008). Additional evidence of attenuated temporal activations during emotion recognition was documented for adults after 12 weeks of LTG monotherapy (Jogia et al., 2008). Once again in subjects with bipolar disorder, Pavluri and 
colleagues detailed augmented activation of lateral temporal, medial frontal, left inferior and middle frontal cortices during a response inhibition task after 14 weeks of initial anti-psychotic treatment followed by LTG monotherapy. These findings corresponded to a normalisation of task-related activations, which correlated with symptom amelioration (Pavuluri et al., 2010).

There is also evidence that LTG may contribute to revert the fMRI correlates associated with ketamine, a well-known antagonist of NMDA glutamate receptors. After administration of ketamine, an investigation reported reduced activation of orbitofrontal and sub-genual cingulate cortices and hyperactivation of posterior cingulate, lateral temporal cortices and thalamus. Interestingly, pre-treatment with LTG led to the suppression of several of the former changes (Deakin et al., 2008).

In summary, several investigations conducted on adolescent and adult patients with mood disorders documented the effect of LTG on disease-relevant regions, largely part of frontolimbic circuits involved in the regulation of emotional responses and/or implicated in response inhibition and task execution. Collectively, such influence was shown to contribute to the restoration of activation patterns described for healthy controls, and hence can be defined as "normalising".

\subsection{GABAPENTIN AND PREGABALIN}

Both gabapentin (GBP) and pregabalin (PGB) selectively bind to the $\alpha_{2} \delta$ subunit of voltagegated calcium channels, leading to enhanced GABA-mediated inhibition and diminished release of neurotransmitters such as glutamate, serotonin, noradrenaline and substance P (Gee et al., 1996; Fink et al., 2002; Dooley et al., 2007). Their clinical indications are particularly broad, ranging from the treatment of focal epilepsy syndromes to neuropathic pain conditions for both compounds, and encompassing generalized anxiety disorder for PGB only (Feltner et al., 2003; Gilron et al., 2005; French et al., 2016). At present, no studies have been conducted to image the action of GBP or PGB in patients with epilepsy. A small number of functional imaging studies, however, addressed the effects of these two drugs in healthy volunteers, investigating brain activity related to pain processing, emotional anticipation and response to emotional faces. With a pharmacological fMRI study design, Iannetti and colleagues elucidated the modulatory effects of a single GBP dose on brain activation during nociceptive mechanical stimulation and experimentally-induced secondary hyperalgesia, the latter being a proxy for 
central sensitization. Although no significant changes were evidenced by pain/hyperalgesiarelated ratings, GBP-correlated imaging features included attenuated activation of operculoinsular cortices in both conditions. During central sensitization, reduced activations were additionally noticed in the brainstem, along with a reduction of stimulus-correlated deactivations during central sensitization only (Iannetti et al., 2005). These findings provided quantitative traits for GBP-correlated effects, suggesting its specific influence on wellestablished pain-processing regions, particularly in the context of central sensitisation. After acute administration of PGB to healthy volunteers, an fMRI paradigm addressing the anticipation responses to positive and negative emotional images reported decreased activation of left amygdala and anterior insula as well as hyperactivation of the anterior cingulate cortex compared with placebo (Aupperle et al., 2011). Moreover, an analysis of emotional face processing in the same study cohort revealed attenuated activation of the left amygdala in relation to fearful face processing, of the left insula specifically for angry faces, and of the fusiform gyrus for stimulus types (Aupperle et al., 2012). Interestingly, reduction of amygdalar activation was also reported in other functional imaging studies addressing the influence of benzodiazepines (Paulus et al., 2005) and selective serotonin reuptake inhibitors during emotional face processing (Arce et al., 2008; Windischberger et al., 2010), while the insula has long been regarded as a key hub for interoception and elaboration of emotional responses (Craig, 2009; Singer et al., 2009). In this context, PGB may be exhibit a similar neural target to other compounds with anxiolytic properties, and exert its effects through a modulation of the activity of limbic and para-limbic circuits with salient roles in regulating responses to emotional stimuli.

\section{INSIGHTS FROM MAGNETIC RESONANCE SPECTROSCOPY}

Proton magnetic resonance spectroscopy $\left({ }^{1} \mathrm{H}-\mathrm{MRS}\right)$ represents a non-invasive technique with the ability to provide quantitative measurements of specific chemical entities in determined volumes of interest. Owing to shifts in resonance frequencies induced by the local proton environment (referring to atoms within and surrounding a given molecule), ${ }^{1} \mathrm{H}-\mathrm{MRS}$ produces a spectrum with several peaks at distinct frequencies, each one specific to a given molecular compound, and whereby the area underlying each peak relates to the concentration of the corresponding molecule (De Graaf, 2013). AEDs are presumed to exert their seizure suppression activity either via potentiating inhibition mechanisms or via an attenuation of 
excitatory neurotransmission (Rogawski and Loscher, 2004), and this represented the underlying rationale of several investigations aiming to determine concentrations of GABA and glutamate (often indistinguishable from glutamine, resulting in a "Glx" composite metric) in patients with epilepsy, and to correlate the former with treatment administration as well as clinical response (Petroff et al., 2000; Mueller et al., 2001; Mueller et al., 2003; Simister et al., 2009). This topic has been elegantly reviewed by Van Veenendaal and collaborators (van Veenendaal et al., 2015), who highlighted the potential of MRS to provide biomarkers predicting AED-related treatment outcomes, and to shed light into the mechanistic underpinnings of treatment failures and cognitive side effects.

AEDs with direct impact on GABAergic neurotransmission represent by far the most frequently investigated by MRS studies. There is a considerable body of evidence indicating enhanced GABA concentrations after acute and chronic administration of vigabatrin (VGB) (Petroff et al., 1996b; Novotny et al., 1999; Petroff et al., 1999; Weber et al., 1999; Mueller et al., 2001; Mueller et al., 2003), an irreversible inhibitor of GABA transaminase, GBP (Petroff et al., 1996c; Kuzniecky et al., 2002; Cai et al., 2012), which increases GABA availability via the modulation of voltage-dependent calcium channels, and TPM (Petroff et al., 2001; Kuzniecky et al., 2002), which potentiates $\mathrm{GABA}_{\mathrm{A}}$-mediated neurotransmission. After treatment initiation with both VGB and GBP, GABA concentrations were shown to correlate with seizure reduction in patients with focal epilepsy (Petroff et al., 1996a; Petroff et al., 1996c). Moreover, VGB doses were shown to correlate linearly with GABA concentrations until dosages of $3 \mathrm{~g} / \mathrm{die}$, exhibiting a plateau for additional dose increases (Petroff et al., 1996d). Mueller and collaborators sought for MRS biomarkers of treatment response in subjects exposed to VGB, and emphasized the relevance of low pre-VGB GABA levels, followed by sharper increases of the latter after treatment start, as a potential response-associated trait in individuals with refractory focal epilepsy (Mueller et al., 2001). Moreover, the difference in GABA concentrations between the epileptogenic and non-epileptogenic hemisphere before VGB treatment was demonstrated to relate to improved seizure control under VGB therapy, regardless of the proximity of data sampling to the epileptogenic focus (Mueller et al., 2003). The above findings are collectively indicative of meaningful correlations between AED administration, modulation of GABA concentrations and clinical improvements. However, such level of detail is available for VGB only, which has unfortunately incurred in marked usage restrictions owing to the risk of potentially irreversible peripheral visual field deficits (Lawden et al., 1999). Exposure-response relationships have not been documented for TPM and GBP, despite evidence of increased GABA concentrations associated with these AEDs. In 
addition, a study investigating the effects of tiagabine, an AED with GABA reuptake inhibition properties, detected no influence on GABA levels after a single-dose administration (Myers $e t$ al., 2014), suggesting of complex and potentially marked compound-specific relationships between drug administration and neurotransmitter effects.

Other MRS studies analysed GABA concentrations associated with AEDs not exhibiting a clearly documented influence on GABAergic neurotransmission. As for LTG, GABA concentrations were not elevated after acute administration nor after 2 weeks of LTG administration, but were shown as enhanced after 4 weeks (Kuzniecky et al., 2002). Similar results were obtained for LEV. A study on healthy volunteers reported no effects on GABA concentrations after three and six hours following a single dose of LEV (Kuzniecky et al., 2008), but subsequent evidence of increased GABA levels 2-6 weeks after initiation of LEV therapy was gathered for the responder subgroup of patients with focal epilepsy (Doelken $e t$ al., 2010). Overall, these findings may suggest that augmented GABAergic neurotransmission be an indirect effect associated with chronic administration of AEDs with non-GABAergic mechanisms of action, and might correlate with response to treatment.

In contrast with the abundance of evidence on GABA levels, there are fewer reports on AEDcorrelated effects on glutamate/glutamine concentrations. Single doses of GBP administered to healthy subjects did not produce measurable glutamate changes (Cai et al., 2012; Preuss et al., 2013), and no modifications of Glx concentrations were detected by a longitudinal study assessing patients with epilepsy treated with VPA (Simister et al., 2007). On the other hand, a study analysing the effects of acute TPM administration in healthy controls, and separately modelling glutamate and glutamine, detected increased glutamine within a sampling voxel located in the anterior cingulate cortex, but no modifications of occipital glutamine and no variations in both anterior cingulate and occipital glutamate (Moore et al., 2006). On balance, no comprehensive inferences can be drawn regarding the influence of various AEDs on excitatory neurotransmission, arguably due to variability in study design and lack of longitudinal assessments.

Other metabolites measured via ${ }^{1} \mathrm{H}$-MRS include $\mathrm{N}$-acetylaspartate (NAA), a widely accepted marker of neuronal integrity (Rigotti et al., 2007), as well as myoinositol, which is a glial marker exhibiting a critical role in the regulation of osmotic balance and, as a precursor of membrane phospholipids and phosphoinositides, is required for cell membrane and myelin integrity (Brand et al., 1993; Haris et al., 2011). In patients with TLE, assessed after one to two years from initiation of AED therapy, lower NAA concentrations within the ipsi- and 
contralateral mesiotemporal sampling voxel were detected in those failing to respond to their first AED compared with those reporting seizure freedom (Campos et al., 2010). These findings may represent a biomarker of AED response, potentially indicating higher levels of neuronal damage in subjects with less favourable profiles of drug-responsiveness. As various AEDs were implicated, however, compound-specific effects could not be established. In a longitudinal study on a mixed epilepsy cohort, mostly represented by idiopathic generalised epilepsy, Simister and colleagues detected reduced myoinositol levels for subjects scanned while on VPA compared with the non-VPA condition, whereas no changes were observed for other metabolites, including Glx and NAA. No correlations were identified between myoinositol levels and seizure frequency or VPA dosage (Simister et al., 2007). Lower concentration of myoinositol was also detected in IGE patients treated with VPA compared with those not taking VPA (Simister et al., 2003). It is tempting to speculate whether altered myoinositol levels associated with VPA exposure may be related to its putative influence on myelination efficiency (Rosenzweig et al., 2012). Animal studies, however, suggest similar effects associated with lithium (O'Donnell et al., 2000), and further research in humans is advocated to establish whether reduction of myoinositol may also occur in association with other AEDs.

With respect to MRS signatures of AED-related cognitive side effects, Van Veenendaal and colleagues recently conducted a cross-sectional study to address the relationship between processing speed and neurotransmitter levels in patient with focal epilepsy receiving AED therapy (van Veenendaal et al., 2016). Across all participants, glutamate concentrations were positively related to processing speed. In individuals taking TPM, deemed as 'high-risk' for cognitive dysfunction, lower glutamate levels and lower processing speed scores were detected compared with patients under LEV and LTG ('low-risk' category). Lower glutamate levels but no differences in processing speed, on the other hand, were observed in the 'intermediate risk' category, grouping VPA, CBZ/OXC and phenytoin, compared to low-risk individuals. Thus, these finding may suggest a potential contribution of AED-associated lower glutamate levels to suboptimal cognitive functioning in patients with epilepsy. However, no correlations were found between GABA levels, cognitive performance and AED-associated risk category. Occipital data sampling might have partially obscured GABA-related effects, in view of previous evidence linking GABA concentrations with motor decision speed in prefrontal, but not occipital areas (Sumner et al., 2010). 
In summary, studies implementing MRS rely on a variety of markers addressing complementary features of brain function, have remarkably shed light on the molecular underpinnings of AED effects, and might provide useful indicators to monitor treatment response and associated side effects.

\section{CONClusion}

Collectively, this review of pharmcao-fMRI and MRS studies strongly supports the potential of imaging investigations to provide MRI biomarkers of AED-correlated effects, which can be characterised within regions and networks implicated in disease pathomechanisms.

The observed effects may not always specific to a given AED in an individual disease entity, given the considerable variability in participant inclusion criteria, study design andspecifics of the implemented paradigms,. A series of highly meaningful patterns however emerge, suggesting the influence of some AEDs on brain targets implicated in disease pathophysiology, or potentially involved in the emergence of drug-related adverse effects:

- TPM has been mostly investigated due to its adverse impact on expressive language and executive functions, and cognitive fMRI studies overall revealed its negative influence on the activation of language areas, accompanied by attenuations of taskcorrelated deactivations.

- CBZ attenuated activation of mesiotemporal activations with a linear dose-response relationship

- LEV favourably influenced the activity in dysfunctional mesiotemporal areas both in TLE and mild cognitive impairment, overall leading to a restoration of normal function.

- VPA's widely variable and often diverging effects warrant further multi-modal MRI analyses on well-characterised cohorts of patients and control subjects, which may allow to better disentangle anatomo-functional trajectories correlated with its usage.

- LTG seems instead to be primarily exerting an effect on fronto-temporo-limbic circuitry involved in the regulation of emotional responses.

- GBP and PGB have been shown to affect areas with prominent roles in nociception and emotion regulation, respectively, in line with their clinical indications. 
It remains to be shown to which extent drugs with distinct pharmacodynamic properties may ultimately influence common final pathways, giving rise to overlapping MRI correlates. This may be the case, for instance, of PGB and other compounds exhibiting anxiolytic properties, such as benzodiazepines and selective serotonin reuptake inhibitors (Aupperle et al., 2011; Aupperle et al., 2012). Further studies are required to understand whether this might also occur for different AEDs in people with epilepsy. A further complicating factor is represented by the intrinsic features of the BOLD contrast utilised in fMRI, which offers an indirect measure of neural activity. As shown for TPM and ZNS, a compound can concurrently exert an influence on both the neuronal and vascular compartments, potentially confounding any mechanistic interpretation of the fMRI correlates of pharmacological interventions. Strategies to disentangle vascular from neuronal effects include the implementation of perfusion MRI sequences such as arterial spin labelling (Borsook et al., 2013), which has been already pursued in some of the above discussed analyses (Aupperle et al., 2011; Aupperle et al., 2012).

While most MRI studies discussed above have either enrolled healthy volunteers, or sought to establish functional markers of drug effects on cognitive networks in patient populations, there is relative scarcity of MRI investigations aiming to establish imaging correlates of drug response, such as seizure-freedom for epilepsy. The latter issue may be more thoroughly addressed via longitudinal imaging studies comparing responders with non-responders and recruiting patients at different time points of their disease course. In this context, multi-modal complementary approaches may undoubtedly represent an asset. As discussed in the previous paragraph, an excellent example is represented by the added value of MRS, which has repeatedly proven useful in establishing the neurochemical underpinnings of the administrations of numerous AEDs, with some reports of correlations between clinical variables and neurotransmitter quantifications. As for any modality, MRS also suffers from limitations, mostly pertaining to data sampling, which is limited to large regions of interest, oftentimes in the occipital lobes only, and does not currently allow distinguishing synaptic from extra-synaptic neurotransmitter pools (Duncan et al., 2014; van Veenendaal et al., 2015). Further insights may come from investigating both imaging and neurophysiological techniques simultaneously, including EEG, TMS, or TMS-EEG, which offer complementary windows into the complex patterns of brain function and have demonstrated considerable potential to provide biomarkers of AED effects (Badawy et al., 2010; Cho et al., 2012; Badawy et al., 2014; Ziemann et al., 2015; Premoli et al., 2016). 
Lastly, the identification of biomarkers associated with pharmacological interventions has gained considerable attention in the context of personalised medicine, whereby inferences pertaining to drug effects should be drawn to tailor clinical decisions to the individual patient. All studies in this review, however, detailed findings obtained at the group level. Whether the above discussed AED-related effects may be utilised for individually customised clinical decision making remains to be shown. To this end, remarkable advancements may be brought about by initiatives aiming to develop personalised brain network models, such as the "Virtual Epileptic Patient" recently proposed by Jirsa and collaborators (Jirsa et al., 2017).

In conclusion, MRI studies have improved our understanding of the impact of AEDs on single regions and complex brain networks, and provide biomarkers of clinical relevance, with encouraging prospects for improving the clinical management of epilepsy.

\section{FUNDING AND CONFLICT OF INTEREST}

LC is funded by a PhD scholarship of the Brain Research Trust, and acknowledges previous support from an Ermenegildo Zegna Founder's Scholarship. FX is funded by the China Scholarship Council, Scholarship No. 201606240215. This work was undertaken at UCLH/UCL who received a proportion of funding from the Department of Health's NIHR Biomedical Research Centre funding scheme.

LC, FX and BW report no conflict of interest. MJK has received consultancy fees from UCB, EISAI, Novartis and GE, outside the submitted work.

\section{ACKNOWLEDGEMENTS}

LC planned the review and prepared the first draft. FX focused on magnetic resonance spectroscopy. BW and MK provided substantial contributions during manuscript revising. All the authors commented on the first draft and on subsequent versions of the review.

\section{REFERENCES}

Biomarkers and surrogate endpoints: preferred definitions and conceptual framework. Clinical pharmacology and therapeutics 2001; 69(3): 89-95. 
Adab N, Kini U, Vinten J, Ayres J, Baker G, Clayton-Smith J, et al. The longer term outcome of children born to mothers with epilepsy. Journal of neurology, neurosurgery, and psychiatry 2004; 75(11): 1575-83.

Ambrosio AF, Soares-Da-Silva P, Carvalho CM, Carvalho AP. Mechanisms of action of carbamazepine and its derivatives, oxcarbazepine, BIA 2-093, and BIA 2-024.

Neurochemical research 2002; 27(1-2): 121-30.

Anticevic A, Repovs G, Shulman GL, Barch DM. When less is more: TPJ and default network deactivation during encoding predicts working memory performance. NeuroImage 2010; 49(3): 2638-48.

Arce E, Simmons AN, Lovero KL, Stein MB, Paulus MP. Escitalopram effects on insula and amygdala BOLD activation during emotional processing. Psychopharmacology 2008; 196(4): 661-72.

Atmaca M, Ozdemir H, Cetinkaya S, Parmaksiz S, Belli H, Poyraz AK, et al. Cingulate gyrus volumetry in drug free bipolar patients and patients treated with valproate or valproate and quetiapine. Journal of psychiatric research 2007; 41(10): 821-7.

Aupperle RL, Ravindran L, Tankersley D, Flagan T, Stein NR, Simmons AN, et al. Pregabalin influences insula and amygdala activation during anticipation of emotional images. Neuropsychopharmacology : official publication of the American College of Neuropsychopharmacology 2011; 36(7): 1466-77.

Aupperle RL, Tankersley D, Ravindran LN, Flagan T, Stein NR, Stein MB, et al. Pregabalin effects on neural response to emotional faces. Frontiers in human neuroscience 2012; 6: 42. Badawy RA, Macdonell RA, Berkovic SF, Newton MR, Jackson GD. Predicting seizure control: cortical excitability and antiepileptic medication. Annals of neurology 2010; 67(1): 64-73.

Badawy RA, Strigaro G, Cantello R. TMS, cortical excitability and epilepsy: the clinical impact. Epilepsy research 2014; 108(2): 153-61.

Bakker A, Albert MS, Krauss G, Speck CL, Gallagher M. Response of the medial temporal lobe network in amnestic mild cognitive impairment to therapeutic intervention assessed by fMRI and memory task performance. NeuroImage Clinical 2015; 7: 688-98.

Bakker A, Krauss GL, Albert MS, Speck CL, Jones LR, Stark CE, et al. Reduction of hippocampal hyperactivity improves cognition in amnestic mild cognitive impairment. Neuron 2012; 74(3): 467-74. 
Bell EC, Willson MC, Wilman AH, Dave S, Silverstone PH. Differential effects of chronic lithium and valproate on brain activation in healthy volunteers. Human psychopharmacology 2005; 20(6): 415-24.

Beltramini GC, Cendes F, Yasuda CL. The effects of antiepileptic drugs on cognitive functional magnetic resonance imaging. Quantitative imaging in medicine and surgery 2015; 5(2): 238-46.

Bernasconi A, Bernasconi N, Bernhardt BC, Schrader D. Advances in MRI for 'cryptogenic' epilepsies. Nature reviews Neurology 2011; 7(2): 99-108.

Bernhardt BC, Bernasconi N, Hong S, Dery S, Bernasconi A. Subregional mesiotemporal network topology is altered in temporal lobe epilepsy. Cereb Cortex 2015; in press.

Bernhardt BC, Hong S, Bernasconi A, Bernasconi N. Imaging structural and functional brain networks in temporal lobe epilepsy. Frontiers in human neuroscience 2013; 7: 624.

Binder JR, Swanson SJ, Hammeke TA, Sabsevitz DS. A comparison of five fMRI protocols for mapping speech comprehension systems. Epilepsia 2008; 49(12): 1980-97.

Biswal BB, Mennes M, Zuo XN, Gohel S, Kelly C, Smith SM, et al. Toward discovery science of human brain function. Proceedings of the National Academy of Sciences of the United States of America 2010; 107(10): 4734-9.

Bootsma HP, Coolen F, Aldenkamp AP, Arends J, Diepman L, Hulsman J, et al. Topiramate in clinical practice: long-term experience in patients with refractory epilepsy referred to a tertiary epilepsy center. Epilepsy \& behavior : E\&B 2004; 5(3): 380-7.

Borsook D, Becerra L, Fava M. Use of functional imaging across clinical phases in CNS drug development. Translational psychiatry 2013; 3: e282.

Borsook D, Becerra L, Hargreaves R. A role for fMRI in optimizing CNS drug development. Nature reviews Drug discovery 2006; 5(5): 411-24.

Brand A, Richter-Landsberg C, Leibfritz D. Multinuclear NMR studies on the energy metabolism of glial and neuronal cells. Developmental neuroscience 1993; 15(3-5): 289-98. Bruhn H, Kleinschmidt A, Boecker H, Merboldt KD, Hanicke W, Frahm J. The effect of acetazolamide on regional cerebral blood oxygenation at rest and under stimulation as assessed by MRI. Journal of cerebral blood flow and metabolism : official journal of the International Society of Cerebral Blood Flow and Metabolism 1994; 14(5): 742-8.

Bullmore E, Sporns O. Complex brain networks: graph theoretical analysis of structural and functional systems. Nature reviews Neuroscience 2009; 10(3): 186-98. 
Caciagli L, Bernhardt BC, Hong SJ, Bernasconi A, Bernasconi N. Functional network alterations and their structural substrate in drug-resistant epilepsy. Frontiers in neuroscience 2014; 8: 411.

Cai K, Nanga RP, Lamprou L, Schinstine C, Elliott M, Hariharan H, et al. The impact of gabapentin administration on brain GABA and glutamate concentrations: a 7T (1)H-MRS study. Neuropsychopharmacology : official publication of the American College of Neuropsychopharmacology 2012; 37(13): 2764-71.

Campos BA, Yasuda CL, Castellano G, Bilevicius E, Li LM, Cendes F. Proton MRS may predict AED response in patients with TLE. Epilepsia 2010; 51(5): 783-8.

Celone KA, Calhoun VD, Dickerson BC, Atri A, Chua EF, Miller SL, et al. Alterations in memory networks in mild cognitive impairment and Alzheimer's disease: an independent component analysis. The Journal of neuroscience : the official journal of the Society for Neuroscience 2006; 26(40): 10222-31.

Chang K, Karchemskiy A, Kelley R, Howe M, Garrett A, Adleman N, et al. Effect of divalproex on brain morphometry, chemistry, and function in youth at high-risk for bipolar disorder: a pilot study. Journal of child and adolescent psychopharmacology 2009; 19(1): 519.

Chang KD, Wagner C, Garrett A, Howe M, Reiss A. A preliminary functional magnetic resonance imaging study of prefrontal-amygdalar activation changes in adolescents with bipolar depression treated with lamotrigine. Bipolar disorders 2008; 10(3): 426-31.

Cheung H, Kamp D, Harris E. An in vitro investigation of the action of lamotrigine on neuronal voltage-activated sodium channels. Epilepsy research 1992; 13(2): 107-12.

Chiang S, Stern JM, Engel J, Jr., Levin HS, Haneef Z. Differences in graph theory functional connectivity in left and right temporal lobe epilepsy. Epilepsy research 2014; 108(10): 177081.

Cho JR, Koo DL, Joo EY, Yoon SM, Ju E, Lee J, et al. Effect of levetiracetam monotherapy on background EEG activity and cognition in drug-naive epilepsy patients. Clinical neurophysiology : official journal of the International Federation of Clinical Neurophysiology 2012; 123(5): 883-91.

Coulter DA. Antiepileptic drug cellular mechanisms of action: where does lamotrigine fit in? Journal of child neurology 1997; 12 Suppl 1: S2-9.

Cousijn H, Rijpkema M, Qin S, van Wingen GA, Fernandez G. Phasic deactivation of the medial temporal lobe enables working memory processing under stress. NeuroImage 2012; 59(2): 1161-7. 
Craig AD. How do you feel--now? The anterior insula and human awareness. Nature reviews Neuroscience 2009; 10(1): 59-70.

Damoiseaux JS, Rombouts SA, Barkhof F, Scheltens P, Stam CJ, Smith SM, et al. Consistent resting-state networks across healthy subjects. Proceedings of the National Academy of Sciences of the United States of America 2006; 103(37): 13848-53.

De Ciantis A, Muti M, Piccolini C, Principi M, Di Renzo A, De Ciantis R, et al. A functional MRI study of language disturbances in subjects with migraine headache during treatment with topiramate. Neurological sciences : official journal of the Italian Neurological Society and of the Italian Society of Clinical Neurophysiology 2008; 29 Suppl 1: S141-3.

De Graaf RA. In vivo NMR spectroscopy: principles and techniques: John Wiley \& Sons; 2013.

Deakin JF, Lees J, McKie S, Hallak JE, Williams SR, Dursun SM. Glutamate and the neural basis of the subjective effects of ketamine: a pharmaco-magnetic resonance imaging study. Archives of general psychiatry 2008; 65(2): 154-64.

Dickerson BC, Salat DH, Bates JF, Atiya M, Killiany RJ, Greve DN, et al. Medial temporal lobe function and structure in mild cognitive impairment. Annals of neurology 2004; 56(1): 27-35.

Doelken MT, Hammen T, Bogner W, Mennecke A, Stadlbauer A, Boettcher U, et al. Alterations of intracerebral gamma-aminobutyric acid (GABA) levels by titration with levetiracetam in patients with focal epilepsies. Epilepsia 2010; 51(8): 1477-82.

Dolcos F, McCarthy G. Brain systems mediating cognitive interference by emotional distraction. The Journal of neuroscience : the official journal of the Society for Neuroscience 2006; 26(7): 2072-9.

Dooley DJ, Taylor CP, Donevan S, Feltner D. Ca2+ channel alpha2delta ligands: novel modulators of neurotransmission. Trends Pharmacol Sci 2007; 28(2): 75-82.

Duncan JS, Winston GP, Koepp MJ, Ourselin S. Brain imaging in the assessment for epilepsy surgery. Lancet neurology 2016; 15(4): 420-33.

Duncan NW, Wiebking C, Northoff G. Associations of regional GABA and glutamate with intrinsic and extrinsic neural activity in humans-a review of multimodal imaging studies. Neurosci Biobehav Rev 2014; 47: 36-52.

Feltner DE, Crockatt JG, Dubovsky SJ, Cohn CK, Shrivastava RK, Targum SD, et al. A randomized, double-blind, placebo-controlled, fixed-dose, multicenter study of pregabalin in patients with generalized anxiety disorder. Journal of clinical psychopharmacology 2003; 23(3): 240-9. 
Fink K, Dooley DJ, Meder WP, Suman-Chauhan N, Duffy S, Clusmann H, et al. Inhibition of neuronal $\mathrm{Ca}(2+)$ influx by gabapentin and pregabalin in the human neocortex.

Neuropharmacology 2002; 42(2): 229-36.

Fleisher AS, Truran D, Mai JT, Langbaum JB, Aisen PS, Cummings JL, et al. Chronic divalproex sodium use and brain atrophy in Alzheimer disease. Neurology 2011; 77(13): 1263-71.

Fox MD, Raichle ME. Spontaneous fluctuations in brain activity observed with functional magnetic resonance imaging. Nature reviews Neuroscience 2007; 8(9): 700-11.

French J, Glue P, Friedman D, Almas M, Yardi N, Knapp L, et al. Adjunctive pregabalin vs gabapentin for focal seizures: Interpretation of comparative outcomes. Neurology 2016; 87(12): 1242-9.

Friston KJ. Functional and effective connectivity: a review. Brain connectivity 2011; 1(1): 13-36.

Gaily E, Kantola-Sorsa E, Hiilesmaa V, Isoaho M, Matila R, Kotila M, et al. Normal intelligence in children with prenatal exposure to carbamazepine. Neurology 2004; 62(1): 2832.

Gee NS, Brown JP, Dissanayake VU, Offord J, Thurlow R, Woodruff GN. The novel anticonvulsant drug, gabapentin (Neurontin), binds to the alpha2delta subunit of a calcium channel. The Journal of biological chemistry 1996; 271(10): 5768-76.

Gilron I, Bailey JM, Tu D, Holden RR, Weaver DF, Houlden RL. Morphine, gabapentin, or their combination for neuropathic pain. The New England journal of medicine 2005; 352(13): 1324-34.

Guerrini R, Belmonte A, Canapicchi R, Casalini C, Perucca E. Reversible pseudoatrophy of the brain and mental deterioration associated with valproate treatment. Epilepsia 1998; 39(1): 27-32.

Hafeman DM, Chang KD, Garrett AS, Sanders EM, Phillips ML. Effects of medication on neuroimaging findings in bipolar disorder: an updated review. Bipolar disorders 2012; 14(4): $375-410$.

Haldane M, Jogia J, Cobb A, Kozuch E, Kumari V, Frangou S. Changes in brain activation during working memory and facial recognition tasks in patients with bipolar disorder with Lamotrigine monotherapy. European neuropsychopharmacology : the journal of the European College of Neuropsychopharmacology 2008; 18(1): 48-54.

Haneef Z, Levin HS, Chiang S. Brain Graph Topology Changes Associated with AntiEpileptic Drug Use. Brain connectivity 2015; 5(5): 284-91. 
Haris M, Cai K, Singh A, Hariharan H, Reddy R. In vivo mapping of brain myo-inositol. NeuroImage 2011; 54(3): 2079-85.

Helmstaedter C, Witt JA. The effects of levetiracetam on cognition: a non-interventional surveillance study. Epilepsy \& behavior : E\&B 2008; 13(4): 642-9.

Helmstaedter C, Witt JA. Cognitive outcome of antiepileptic treatment with levetiracetam versus carbamazepine monotherapy: a non-interventional surveillance trial. Epilepsy \& behavior : E\&B 2010; 18(1-2): 74-80.

Hermann B, Seidenberg M, Jones J. The neurobehavioural comorbidities of epilepsy: can a natural history be developed? Lancet neurology 2008; 7(2): 151-60.

Honey CJ, Kotter R, Breakspear M, Sporns O. Network structure of cerebral cortex shapes functional connectivity on multiple time scales. Proceedings of the National Academy of Sciences of the United States of America 2007; 104(24): 10240-5.

Iannetti GD, Zambreanu L, Wise RG, Buchanan TJ, Huggins JP, Smart TS, et al.

Pharmacological modulation of pain-related brain activity during normal and central sensitization states in humans. Proceedings of the National Academy of Sciences of the United States of America 2005; 102(50): 18195-200.

Jansen JF, Aldenkamp AP, Marian Majoie HJ, Reijs RP, de Krom MC, Hofman PA, et al. Functional MRI reveals declined prefrontal cortex activation in patients with epilepsy on topiramate therapy. Epilepsy \& behavior : E\&B 2006; 9(1): 181-5.

Jirsa VK, Proix T, Perdikis D, Woodman MM, Wang H, Gonzalez-Martinez J, et al. The Virtual Epileptic Patient: Individualized whole-brain models of epilepsy spread. NeuroImage 2017; 145(Pt B): 377-88.

Jogia J, Haldane M, Cobb A, Kumari V, Frangou S. Pilot investigation of the changes in cortical activation during facial affect recognition with lamotrigine monotherapy in bipolar disorder. The British journal of psychiatry : the journal of mental science 2008; 192(3): 197201.

Jokeit H, Okujava M, Woermann FG. Carbamazepine reduces memory induced activation of mesial temporal lobe structures: a pharmacological fMRI-study. BMC neurology 2001; 1: 6. Ketter TA, Manji HK, Post RM. Potential mechanisms of action of lamotrigine in the treatment of bipolar disorders. Journal of clinical psychopharmacology 2003; 23(5): 484-95. Kim H. Neural activity that predicts subsequent memory and forgetting: a meta-analysis of 74 fMRI studies. NeuroImage 2011; 54(3): 2446-61.

Koepp MJ. The help of biomarkers in the prevention of epilepsy. Lancet neurology 2016; 15(8): 782-4. 
Koepp MJ, Caciagli L, Pressler RM, Lehnertz K, Beniczky S. Reflex seizures, traits, and epilepsies: from physiology to pathology. Lancet neurology 2016; 15(1): $92-105$.

Kupferschmidt DA, Zakzanis KK. Toward a functional neuroanatomical signature of bipolar disorder: quantitative evidence from the neuroimaging literature. Psychiatry research 2011; 193(2): 71-9.

Kuzniecky R, Ho S, Pan J, Martin R, Gilliam F, Faught E, et al. Modulation of cerebral GABA by topiramate, lamotrigine, and gabapentin in healthy adults. Neurology 2002; 58(3): 368-72.

Kuzniecky R, Pan J, Burns A, Devinsky O, Hetherington H. Levetiracetam has no acute effects on brain gamma-aminobutyric acid levels. Epilepsy \& behavior : E\&B 2008; 12(2): $242-4$.

Kwan P, Arzimanoglou A, Berg AT, Brodie MJ, Allen Hauser W, Mathern G, et al. Definition of drug resistant epilepsy: consensus proposal by the ad hoc Task Force of the ILAE Commission on Therapeutic Strategies. Epilepsia 2010; 51(6): 1069-77.

Kwon OY, Park SP. Zonisamide decreases current-source density of high Beta frequency of electroencephalogram. Journal of epilepsy research 2013; 3(2): 63-9.

Lawden MC, Eke T, Degg C, Harding GF, Wild JM. Visual field defects associated with vigabatrin therapy. Journal of neurology, neurosurgery, and psychiatry 1999; 67(6): 716-22. Lee S, Sziklas V, Andermann F, Farnham S, Risse G, Gustafson M, et al. The effects of adjunctive topiramate on cognitive function in patients with epilepsy. Epilepsia 2003; 44(3): $339-47$.

Leniger T, Thone J, Wiemann M. Topiramate modulates $\mathrm{pH}$ of hippocampal CA3 neurons by combined effects on carbonic anhydrase and Cl-/HCO3- exchange. British journal of pharmacology 2004; 142(5): 831-42.

Leppik IE. Zonisamide: chemistry, mechanism of action, and pharmacokinetics. Seizure : the journal of the British Epilepsy Association 2004; 13 Suppl 1: S5-9; discussion S10. Li X, Large CH, Ricci R, Taylor JJ, Nahas Z, Bohning DE, et al. Using interleaved transcranial magnetic stimulation/functional magnetic resonance imaging (fMRI) and dynamic causal modeling to understand the discrete circuit specific changes of medications: lamotrigine and valproic acid changes in motor or prefrontal effective connectivity. Psychiatry research 2011; 194(2): 141-8.

Li X, Ricci R, Large CH, Anderson B, Nahas Z, Bohning DE, et al. Interleaved transcranial magnetic stimulation and fMRI suggests that lamotrigine and valproic acid have different effects on corticolimbic activity. Psychopharmacology 2010; 209(3): 233-44. 
Li X, Teneback CC, Nahas Z, Kozel FA, Large C, Cohn J, et al. Interleaved transcranial magnetic stimulation/functional MRI confirms that lamotrigine inhibits cortical excitability in healthy young men. Neuropsychopharmacology : official publication of the American College of Neuropsychopharmacology 2004; 29(7): 1395-407.

Liao W, Zhang Z, Pan Z, Mantini D, Ding J, Duan X, et al. Altered functional connectivity and small-world in mesial temporal lobe epilepsy. PloS one 2010; 5(1): e8525.

Liu L, Zheng T, Morris MJ, Wallengren C, Clarke AL, Reid CA, et al. The mechanism of carbamazepine aggravation of absence seizures. The Journal of pharmacology and experimental therapeutics 2006; 319(2): 790-8.

Logothetis NK, Pauls J, Augath M, Trinath T, Oeltermann A. Neurophysiological investigation of the basis of the fMRI signal. Nature 2001; 412(6843): 150-7.

Loscher W. Basic pharmacology of valproate: a review after 35 years of clinical use for the treatment of epilepsy. CNS drugs 2002; 16(10): 669-94.

Lynch BA, Lambeng N, Nocka K, Kensel-Hammes P, Bajjalieh SM, Matagne A, et al. The synaptic vesicle protein SV2A is the binding site for the antiepileptic drug levetiracetam.

Proceedings of the National Academy of Sciences of the United States of America 2004; 101(26): 9861-6.

Martin R, Kuzniecky R, Ho S, Hetherington H, Pan J, Sinclair K, et al. Cognitive effects of topiramate, gabapentin, and lamotrigine in healthy young adults. Neurology 1999; 52(2): $321-7$.

Meador KJ, Baker GA, Browning N, Clayton-Smith J, Combs-Cantrell DT, Cohen M, et al. Cognitive function at 3 years of age after fetal exposure to antiepileptic drugs. The New England journal of medicine 2009; 360(16): 1597-605.

Meador KJ, Baker GA, Browning N, Cohen MJ, Bromley RL, Clayton-Smith J, et al. Fetal antiepileptic drug exposure and cognitive outcomes at age 6 years (NEAD study): a prospective observational study. Lancet neurology 2013; 12(3): 244-52.

Meador KJ, Baker GA, Browning N, Cohen MJ, Clayton-Smith J, Kalayjian LA, et al. Foetal antiepileptic drug exposure and verbal versus non-verbal abilities at three years of age. Brain : a journal of neurology 2011; 134(Pt 2): 396-404.

Meador KJ, Gevins A, Loring DW, McEvoy LK, Ray PG, Smith ME, et al.

Neuropsychological and neurophysiologic effects of carbamazepine and levetiracetam.

Neurology 2007; 69(22): 2076-84.

Meador KJ, Loring DW, Hulihan JF, Kamin M, Karim R. Differential cognitive and behavioral effects of topiramate and valproate. Neurology 2003; 60(9): 1483-8. 
Meador KJ, Loring DW, Vahle VJ, Ray PG, Werz MA, Fessler AJ, et al. Cognitive and behavioral effects of lamotrigine and topiramate in healthy volunteers. Neurology 2005; 64(12): 2108-14.

Mecarelli O, Vicenzini E, Pulitano P, Vanacore N, Romolo FS, Di Piero V, et al. Clinical, cognitive, and neurophysiologic correlates of short-term treatment with carbamazepine, oxcarbazepine, and levetiracetam in healthy volunteers. The Annals of pharmacotherapy 2004; 38(11): 1816-22.

Mehta MA, O'Daly OG. Pharmacological application of fMRI. Methods Mol Biol 2011; 711: $551-65$.

Moore CM, Wardrop M, de BFB, Renshaw PF. Topiramate raises anterior cingulate cortex glutamine levels in healthy men; a 4.0 T magnetic resonance spectroscopy study. Psychopharmacology 2006; 188(2): 236-43.

Mueller SG, Weber OM, Duc CO, Meier D, Russ W, Boesiger P, et al. Effects of vigabatrin on brain $\mathrm{GABA}+/ \mathrm{Cr}$ signals in focus-distant and focus-near brain regions monitored by $1 \mathrm{H}-$ NMR spectroscopy. European journal of neurology : the official journal of the European Federation of Neurological Societies 2003; 10(1): 45-52.

Mueller SG, Weber OM, Duc CO, Weber B, Meier D, Russ W, et al. Effects of vigabatrin on brain GABA+/CR signals in patients with epilepsy monitored by 1H-NMR-spectroscopy: responder characteristics. Epilepsia 2001; 42(1): 29-40.

Mula M. Topiramate and cognitive impairment: evidence and clinical implications.

Therapeutic advances in drug safety 2012; 3(6): 279-89.

Mula M, Trimble MR. Antiepileptic drug-induced cognitive adverse effects: potential mechanisms and contributing factors. CNS drugs 2009; 23(2): 121-37.

Mula M, Trimble MR, Thompson P, Sander JW. Topiramate and word-finding difficulties in patients with epilepsy. Neurology 2003; 60(7): 1104-7.

Myers JF, Evans CJ, Kalk NJ, Edden RA, Lingford-Hughes AR. Measurement of GABA using J-difference edited 1H-MRS following modulation of synaptic GABA concentration with tiagabine. Synapse 2014; 68(8): 355-62.

Nadebaum C, Anderson VA, Vajda F, Reutens DC, Barton S, Wood AG. Language skills of school-aged children prenatally exposed to antiepileptic drugs. Neurology 2011; 76(8): 71926.

Nalivaeva NN, Belyaev ND, Turner AJ. Sodium valproate: an old drug with new roles. Trends Pharmacol Sci 2009; 30(10): 509-14. 
Nathan PJ, Phan KL, Harmer CJ, Mehta MA, Bullmore ET. Increasing pharmacological knowledge about human neurological and psychiatric disorders through functional neuroimaging and its application in drug discovery. Current opinion in pharmacology 2014; 14: 54-61.

Novotny EJ, Jr., Hyder F, Shevell M, Rothman DL. GABA changes with vigabatrin in the developing human brain. Epilepsia 1999; 40(4): 462-6.

O'Donnell T, Rotzinger S, Nakashima TT, Hanstock CC, Ulrich M, Silverstone PH. Chronic lithium and sodium valproate both decrease the concentration of myo-inositol and increase the concentration of inositol monophosphates in rat brain. Brain research 2000; 880(1-2): 8491.

Ogawa S, Lee TM, Kay AR, Tank DW. Brain magnetic resonance imaging with contrast dependent on blood oxygenation. Proc Natl Acad Sci USA 1990; 87(24): 9868-72.

Ojemann LM, Ojemann GA, Dodrill CB, Crawford CA, Holmes MD, Dudley DL. Language Disturbances as Side Effects of Topiramate and Zonisamide Therapy. Epilepsy \& behavior : E\&B 2001; 2(6): 579-84.

Ortinski P, Meador KJ. Cognitive side effects of antiepileptic drugs. Epilepsy \& behavior : E\&B 2004; 5 Suppl 1: S60-5.

Owen AM, McMillan KM, Laird AR, Bullmore E. N-back working memory paradigm: a meta-analysis of normative functional neuroimaging studies. Human brain mapping 2005; 25(1): 46-59.

Papazian O, Canizales E, Alfonso I, Archila R, Duchowny M, Aicardi J. Reversible dementia and apparent brain atrophy during valproate therapy. Annals of neurology 1995; 38(4): 68791.

Pardoe HR, Berg AT, Jackson GD. Sodium valproate use is associated with reduced parietal lobe thickness and brain volume. Neurology 2013; 80(20): 1895-900.

Patsalos PN. Properties of antiepileptic drugs in the treatment of idiopathic generalized epilepsies. Epilepsia 2005; 46 Suppl 9: 140-8.

Paulus MP, Feinstein JS, Castillo G, Simmons AN, Stein MB. Dose-dependent decrease of activation in bilateral amygdala and insula by lorazepam during emotion processing. Archives of general psychiatry 2005; 62(3): 282-8.

Pavuluri MN, Passarotti AM, Harral EM, Sweeney JA. Enhanced prefrontal function with pharmacotherapy on a response inhibition task in adolescent bipolar disorder. The Journal of clinical psychiatry 2010; 71(11): 1526-34. 
Perucca E. A pharmacological and clinical review on topiramate, a new antiepileptic drug. Pharmacological research : the official journal of the Italian Pharmacological Society 1997; 35(4): 241-56.

Perucca E. Pharmacological and therapeutic properties of valproate: a summary after 35 years of clinical experience. CNS drugs 2002; 16(10): 695-714.

Perucca P, Gilliam FG. Adverse effects of antiepileptic drugs. Lancet neurology 2012; 11(9): 792-802.

Petroff OA, Behar KL, Mattson RH, Rothman DL. Human brain gamma-aminobutyric acid levels and seizure control following initiation of vigabatrin therapy. Journal of neurochemistry 1996a; 67(6): 2399-404.

Petroff OA, Hyder F, Collins T, Mattson RH, Rothman DL. Acute effects of vigabatrin on brain GABA and homocarnosine in patients with complex partial seizures. Epilepsia 1999; 40(7): 958-64.

Petroff OA, Hyder F, Rothman DL, Mattson RH. Topiramate rapidly raises brain GABA in epilepsy patients. Epilepsia 2001; 42(4): 543-8.

Petroff OA, Mattson RH, Rothman DL. Proton MRS: GABA and glutamate. AdvNeurol 2000; 83: 261-71.

Petroff OA, Rothman DL, Behar KL, Collins TL, Mattson RH. Human brain GABA levels rise rapidly after initiation of vigabatrin therapy. Neurology 1996b; 47(6): 1567-71. Petroff OA, Rothman DL, Behar KL, Lamoureux D, Mattson RH. The effect of gabapentin on brain gamma-aminobutyric acid in patients with epilepsy. Annals of neurology 1996c; 39(1): 95-9.

Petroff OA, Rothman DL, Behar KL, Mattson RH. Human brain GABA levels rise after initiation of vigabatrin therapy but fail to rise further with increasing dose. Neurology 1996d; 46(5): 1459-63.

Piazzini A, Chifari R, Canevini MP, Turner K, Fontana SP, Canger R. Levetiracetam: an improvement of attention and of oral fluency in patients with partial epilepsy. Epilepsy research 2006; 68(3): 181-8.

Pizzagalli DA. Frontocingulate dysfunction in depression: toward biomarkers of treatment response. Neuropsychopharmacology : official publication of the American College of Neuropsychopharmacology 2011; 36(1): 183-206.

Premoli I, Biondi A, Carlesso S, Rivolta D, Richardson MP. Lamotrigine and levetiracetam exert a similar modulation of TMS-evoked EEG potentials. Epilepsia 2016. 
Preuss N, van der Veen JW, Carlson PJ, Shen J, Hasler G. Low single dose gabapentin does not affect prefrontal and occipital gamma-aminobutyric acid concentrations. European neuropsychopharmacology : the journal of the European College of Neuropsychopharmacology 2013; 23(12): 1708-13.

Price CJ. A review and synthesis of the first 20 years of PET and fMRI studies of heard speech, spoken language and reading. NeuroImage 2012; 62(2): 816-47.

Rao SM, Binder JR, Bandettini PA, Hammeke TA, Yetkin FZ, Jesmanowicz A, et al. Functional magnetic resonance imaging of complex human movements. Neurology 1993; 43(11): 2311-8.

Rigotti DJ, Inglese M, Gonen O. Whole-brain N-acetylaspartate as a surrogate marker of neuronal damage in diffuse neurologic disorders. AJNR American journal of neuroradiology 2007; 28(10): 1843-9.

Rogawski MA, Loscher W. The neurobiology of antiepileptic drugs. Nature reviews Neuroscience 2004; 5(7): 553-64.

Rosenberg G. The mechanisms of action of valproate in neuropsychiatric disorders: can we see the forest for the trees? Cellular and molecular life sciences : CMLS 2007; 64(16): 2090103.

Rosenzweig I, Vukadinovic Z, Turner AJ, Catani M. Neuroconnectivity and valproic acid: the myelin hypothesis. Neurosci Biobehav Rev 2012; 36(8): 1848-56.

Sander JW. The epidemiology of epilepsy revisited. Current opinion in neurology 2003; 16(2): $165-70$.

Schlosser RG, Wagner G, Koch K, Dahnke R, Reichenbach JR, Sauer H. Fronto-cingulate effective connectivity in major depression: a study with fMRI and dynamic causal modeling. NeuroImage 2008; 43(3): 645-55.

Shank RP, Gardocki JF, Vaught JL, Davis CB, Schupsky JJ, Raffa RB, et al. Topiramate: preclinical evaluation of structurally novel anticonvulsant. Epilepsia 1994; 35(2): 450-60. Sheline YI, Price JL, Yan Z, Mintun MA. Resting-state functional MRI in depression unmasks increased connectivity between networks via the dorsal nexus. Proceedings of the National Academy of Sciences of the United States of America 2010; 107(24): 11020-5. Shen S, Sandoval J, Swiss VA, Li J, Dupree J, Franklin RJ, et al. Age-dependent epigenetic control of differentiation inhibitors is critical for remyelination efficiency. Nature neuroscience 2008; 11(9): 1024-34.

Simister RJ, McLean MA, Barker GJ, Duncan JS. Proton MRS reveals frontal lobe metabolite abnormalities in idiopathic generalized epilepsy. Neurology 2003; 61(7): 897-902. 
Simister RJ, McLean MA, Barker GJ, Duncan JS. The effect of sodium valproate on proton MRS visible neurochemical concentrations. Epilepsy research 2007; 74(2-3): 215-9.

Simister RJ, McLean MA, Barker GJ, Duncan JS. Proton MR spectroscopy of metabolite concentrations in temporal lobe epilepsy and effect of temporal lobe resection. Epilepsy research 2009; 83(2-3): 168-76.

Singer T, Critchley HD, Preuschoff K. A common role of insula in feelings, empathy and uncertainty. Trends in cognitive sciences 2009; 13(8): 334-40.

Sonuga-Barke EJ, Castellanos FX. Spontaneous attentional fluctuations in impaired states and pathological conditions: a neurobiological hypothesis. Neurosci Biobehav Rev 2007; 31(7): 977-86.

Sporns O. From simple graphs to the connectome: networks in neuroimaging. NeuroImage 2012; 62(2): 881-6.

Strakowski SM, Adler CM, Almeida J, Altshuler LL, Blumberg HP, Chang KD, et al. The functional neuroanatomy of bipolar disorder: a consensus model. Bipolar disorders 2012; 14(4): $313-25$.

Stretton J, Winston G, Sidhu M, Centeno M, Vollmar C, Bonelli S, et al. Neural correlates of working memory in Temporal Lobe Epilepsy--an fMRI study. NeuroImage 2012; 60(3): 1696-703.

Stretton J, Winston GP, Sidhu M, Bonelli S, Centeno M, Vollmar C, et al. Disrupted segregation of working memory networks in temporal lobe epilepsy. NeuroImage Clinical 2013; 2: 273-81.

Sumner P, Edden RA, Bompas A, Evans CJ, Singh KD. More GABA, less distraction: a neurochemical predictor of motor decision speed. Nature neuroscience 2010; 13(7): 825-7. Szaflarski JP, Allendorfer JB. Topiramate and its effect on fMRI of language in patients with right or left temporal lobe epilepsy. Epilepsy \& behavior : E\&B 2012; 24(1): 74-80.

Tang Y, Xia W, Yu X, Zhou B, Wu X, Lui S, et al. Altered cerebral activity associated with topiramate and its withdrawal in patients with epilepsy with language impairment: An fMRI study using the verb generation task. Epilepsy \& behavior : E\&B 2016; 59: 98-104.

Tariot PN, Loy R, Ryan JM, Porsteinsson A, Ismail S. Mood stabilizers in Alzheimer's disease: symptomatic and neuroprotective rationales. Advanced drug delivery reviews 2002; 54(12): 1567-77.

Tariot PN, Schneider LS, Cummings J, Thomas RG, Raman R, Jakimovich LJ, et al. Chronic divalproex sodium to attenuate agitation and clinical progression of Alzheimer disease. Archives of general psychiatry 2011; 68(8): 853-61. 
Thompson PJ, Baxendale SA, Duncan JS, Sander JW. Effects of topiramate on cognitive function. Journal of neurology, neurosurgery, and psychiatry 2000; 69(5): 636-41.

Thompson PJ, Trimble MR. Sodium valproate and cognitive functioning in normal volunteers. British journal of clinical pharmacology 1981; 12(6): 819-24.

van Veenendaal TM, DM IJ, Aldenkamp AP, Hofman PA, Vlooswijk MC, Rouhl RP, et al. Metabolic and functional MR biomarkers of antiepileptic drug effectiveness: A review.

Neurosci Biobehav Rev 2015; 59: 92-9.

van Veenendaal TM, DM IJ, Aldenkamp AP, Lazeron RH, Puts NA, Edden RA, et al. Glutamate concentrations vary with antiepileptic drug use and mental slowing. Epilepsy \& behavior : E\&B 2016; 64(Pt A): 200-5.

Vollmar C, O'Muircheartaigh J, Barker GJ, Symms MR, Thompson P, Kumari V, et al. Motor system hyperconnectivity in juvenile myoclonic epilepsy: a cognitive functional magnetic resonance imaging study. Brain : a journal of neurology 2011; 134(Pt 6): 1710-9. Vollmar C, O'Muircheartaigh J, Symms MR, Barker GJ, Thompson P, Kumari V, et al. Altered microstructural connectivity in juvenile myoclonic epilepsy: the missing link. Neurology 2012; 78(20): 1555 - 9.

Wandschneider B, Koepp MJ. Pharmaco fMRI: Determining the functional anatomy of the effects of medication. NeuroImage Clinical 2016; 12: 691-7.

Wandschneider B, Stretton J, Sidhu M, Centeno M, Kozak LR, Symms M, et al. Levetiracetam reduces abnormal network activations in temporal lobe epilepsy. Neurology 2014; 83(17): 1508-12.

Wandschneider B, Thompson PJ, Vollmar C, Koepp MJ. Frontal lobe function and structure in juvenile myoclonic epilepsy: a comprehensive review of neuropsychological and imaging data. Epilepsia 2012; 53(12): 2091-8.

Wang J, Qiu S, Xu Y, Liu Z, Wen X, Hu X, et al. Graph theoretical analysis reveals disrupted topological properties of whole brain functional networks in temporal lobe epilepsy. Clinical neurophysiology : official journal of the International Federation of Clinical Neurophysiology 2014.

Weber OM, Verhagen A, Duc CO, Meier D, Leenders KL, Boesiger P. Effects of vigabatrin intake on brain GABA activity as monitored by spectrally edited magnetic resonance spectroscopy and positron emission tomography. Magn ResonImaging 1999; 17(3): 417-25. Weissman DH, Roberts KC, Visscher KM, Woldorff MG. The neural bases of momentary lapses in attention. Nature neuroscience 2006; 9(7): 971-8. 
Windischberger C, Lanzenberger R, Holik A, Spindelegger C, Stein P, Moser U, et al. Areaspecific modulation of neural activation comparing escitalopram and citalopram revealed by pharmaco-fMRI: a randomized cross-over study. NeuroImage 2010; 49(2): 1161-70.

Wolf P, Yacubian EM, Avanzini G, Sander T, Schmitz B, Wandschneider B, et al. Juvenile myoclonic epilepsy: A system disorder of the brain. Epilepsy research 2015; 114: 2-12.

Wood AG, Chen J, Barton S, Nadebaum C, Anderson VA, Catroppa C, et al. Altered cortical thickness following prenatal sodium valproate exposure. Ann Clin Transl Neurol 2014; 1(7): 497-501.

Yasuda CL, Centeno M, Vollmar C, Stretton J, Symms M, Cendes F, et al. The effect of topiramate on cognitive fMRI. Epilepsy research 2013; 105(1-2): 250-5.

Zang Y, Jiang T, Lu Y, He Y, Tian L. Regional homogeneity approach to fMRI data analysis. NeuroImage 2004; 22(1): 394-400.

Zhang Q, Yang F, Hu Z, Zhang Z, Xu Q, Dante M, et al. Resting-state fMRI revealed different brain activities responding to valproic acid and levetiracetam in benign epilepsy with central-temporal spikes. European radiology 2016.

Ziemann U, Reis J, Schwenkreis P, Rosanova M, Strafella A, Badawy R, et al. TMS and drugs revisited 2014. Clinical neurophysiology : official journal of the International Federation of Clinical Neurophysiology 2015; 126(10): 1847-68. 